\title{
Genome-Wide Allelic Status
}

National Cancer Institute

\section{Source}

National Cancer Institute. Genome-Wide Allelic Status. NCI Thesaurus. Code C129902.

The analysis of all of the alleles present in the genome in a biological sample. 